\title{
Die Knochen bleiben stabil
}

Fragestellung: Sind bei depressiven Patienten im jungen und mittleren Lebensalter Knochendichte und relevante biochemische Parameter des Knochenstoffwechsels reduziert?

Hintergrund: Viele Studien zeigten einen Zusammenhang zwischen klinisch relevanter Depression (Major Depressive Disorder, MDD) und Reduktion der Knochendichte mit der Folge eines erhöhten Osteoporoserisikos. Zum Pathomechanismus kann gegenwärtig nur spekuliert werden, wobei ungünstiger Lebensstil (Bewegungsmangel, einseitige Ernährung), antidepressive Medikation (vor allem selektive Serotonin-Wiederaufnahme-Hemmer, SSRI) und verschiedene neuroendokrinologische oder immunologische Veränderungen angeschuldigt werden.

Patienten und Methodik: Bei MDD-Patienten im jungen und mittleren Erwachsenenalter, die im Rahmen einer multizentrischen Studie rekrutiert wurden, erfolgten Knochendichtemessungen (mittels Dual-Rönt-

Malik P, Gasser RW, Moncayo $\mathrm{RC}$ et al. Bone mineral density and bone metabolism in patients with major depressive disorder without somatic comorbidities. Prog Neuropsychopharmacol Biol Psychiatry 2013; 44: 58-63 gen-Absorptiometrie) im Bereich der Lendenwirbelsäule (LWS) und des Schenkelhalses. Neben dem Depressionsschweregrad (MontgomeryÅsberg Depression Rating Scale, MADRS) wurde eine Vielzahl biochemischer, endokrinologischer und immu- nologischer Marker mit Relevanz für den Knochenstoffwechsel bestimmt. Wichtiges Ausschlusskriterium waren internistische Erkrankungen und Medikamente mit potenzieller negativer Auswirkung auf den Knochenstoffwechsel (Ausnahme: Antidepressiva) und bei Frauen Menopause.

Ergebnisse: Nach Ausschluss von 104 Patienten aufgrund von Komorbiditäten wurden 50 Patienten analysiert. Der durchschnittliche Depressionsschweregrad war hoch (MADRS-Score 27,5) und auch die Erkrankungsdauer war im Schnitt eher lang (4,9 Jahre). Mit einer Ausnahme zeigte sich bei keinem Teilnehmer eine relevant erniedrigte Knochendichte (definiert als zWert $\leq-2,0)$. Knochendichteminderung korrelierte weder mit dem Schweregrad noch mit der Dauer der Depression. Die osteoklastischen Parameter Osteocalcin und $\beta$-CrossLaps lagen überwiegend im Normbereich. Der Wert für das knochenprotektive Osteoprotegerin (OPG) war nur bei wenigen Patienten erhältlich und im Schnitt sogar leicht erhöht. 74,5\% der Patienten wiesen einen erniedrigten 25-OH-Vitamin-D3-Spiegel auf. Die SSRI-Einnahme hatte keinen Einfluss auf die Knochendichte, während Rauchen mit erniedrigter Knochendichte assoziiert war.

Schlussfolgerungen: Somatisch gesunde, akut depressive Patienten im jüngeren und mittleren Lebensalter zeigen keine Osteopenie oder erhöhte osteoklastische Aktivität. Auch die SSRI-Einnahme führt in dieser Patientengruppe nicht zu einer Knochendichteminderung.

\section{-Kommentar von Christian Lange-Asschenfeldt und Florence Hellen, Düsseldorf}

\section{Depression ist kein unabhängiger Risikofaktor}

Zahlreiche Studien haben auf eine Reduktion der Knochendichte oder Erhöhung biochemischer osteoklastischer Parameter bei depressiven Störungen hingewiesen [1], jedoch existieren bei sehr heterogenen Studienpopulationen zahlreiche Einflussfaktoren: Neben Alter, Geschlecht und Hormonstatus sind dies vor allem risikofördernde Medikation, somatische und psychiatrische Begleiterkrankungen, Nikotin- und Alkoholkonsum, Bewegungsmangel, ungünstige Ernährung, Adipositas und VitaminD-Mangel [2]. Die vorliegende Studie ist vergleichsweise klein und verfügt über keine Kontrollgruppe, jedoch ist ihr Verdienst die weitgehende Bereinigung solcher konfundierender Faktoren durch strenge Ausschlusskriterien. Bei ausreichendem Symptomschweregrad und auch genügend langer Krankheitsdauer erbrachte sie unter diesen Voraussetzungen ein negatives Ergebnis. In der hier referierten Studie ging als zweites Hauptergebnis auch die SSRI-Behandlung nicht mit einer verminderten Knochendichte oder erhöhten Knochenabbaumarkern einher. Auch diesbezügliche Daten sind bisher nicht eindeutig trotz eines Überwiegens positiver Studien [3]. Zusammengefasst dürfte bei depressiven Patienten das kumulative Risiko mehrerer
Faktoren ausschlaggebend für eine Osteopenie sein. Die MDD oder eine SSRI-Behandlung sind möglicherweise keine unabhängigen Risikofaktoren.
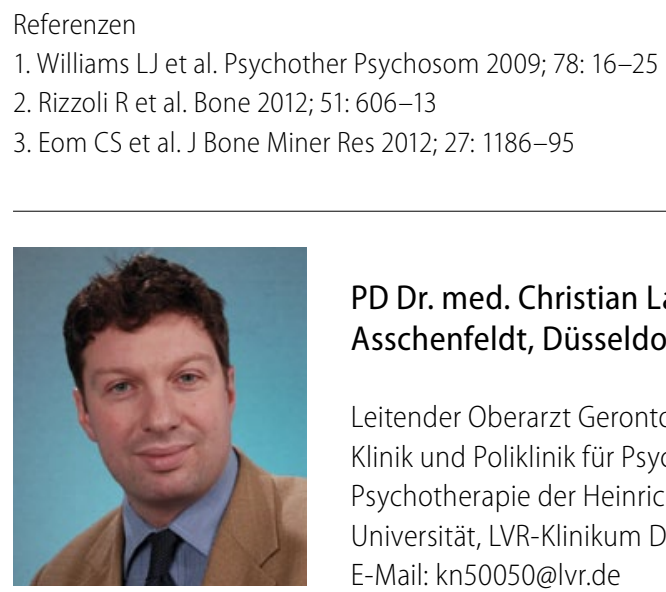

PD Dr. med. Christian Lange-

Asschenfeldt, Düsseldorf

Leitender Oberarzt Gerontopsychiatrie, Klinik und Poliklinik für Psychiatrie und Psychotherapie der Heinrich-HeineUniversität, LVR-Klinikum Düsseldorf E-Mail:kn50050@Ivr.de 